\title{
Alcohol Consumption Promotes the Invasion and Proliferation of Gastric Cancer
}

\author{
Jie Ding ${ }^{\#}$, Hao Wang ${ }^{\#}$, Xing Kang, Feng Wang, Hong Chen, Min Feng, Wen Xian Guan \\ Department of General Surgery, Nanjing Drum Tower Hospital, The Affiliated Hospital of Nanjing University Medical School, \\ Nanjing, Jiangsu 210008, China
}

\begin{abstract}
Objective To determine the effects of alcohol on the invasion and proliferation of gastric cancer. Methods In an in vitro study, gastric cancer cells were treated with different concentrations of alcohol. The protein levels of matrix metalloproteinase (MMP)2/7/9/13, Cyclin D and PCNA were determined by Western blot. The transcription levels of MMP2/7/9/13 were determined by qRT-PCR. An MMPs inhibitor (GM6001) was used to analyze the role of MMPs in the invasion of gastric cancer cells. In an in vivo experiment, the effects of alcohol on the proliferation of gastric cancer was analyzed in a heterotopic transplantation animal tumor model. Results The protein levels of MMP2/7/9/13 were increased in gastric cancer cells (AGS and HGC-27) compared with normal gastric epithelial cells (GES). Alcohol treatment resulted in further elevation of the MMPs expression in the cancer cells and promoted the invasion of the gastric cancer cells. GM6001 inhibited these effects. In addition, alcohol treatment promoted the expression of Cyclin D and PCNA in cancer cells and gastric tumor proliferation in the animal model. Conclusion Alcohol consumption promoted the invasion and proliferation of gastric cancer. Confirmation should be obtained in further studies in additional models.
\end{abstract}

Key words: Gastric cancer; Alcohol; Invasion; Proliferation; Matrix metalloproteinases

\section{Introduction}

Cancer is a leading cause of human death, and produces serious economic burdens. An epidemiological study published in 2018 provided a status report on the global burden of 36 cancers in 185 countries. The incidence of gastric cancer ranked fifth in the world, and was third for cancer-related mortality [1]. In China, gastric cancer is the second most common cancer and the third leading cause of death from malignant disease [2].

Alcohol is one of the most commonly and regularly used chemical substances. The stomach is one of the major target organs of alcohol. Epidemiological studies indicated that alcohol increases both the incidence and mortality of gastric cancer $[3,4]$. However, the exact effects of alcohol on gastric cancer and the underlying mechanism (s) by which alcohol promotes gastric cancer development are still unclear.

Degradation of the extracellular matrix (ECM) is an important step in tumor metastasis [5]. Extracellular matrixdegrading enzymes, such as matrix metalloproteinases

\footnotetext{
\#Jie Ding and Hao Wang made an equal contribution to this paper. Corresponding author: Wen Xian Guan, MD, PhD, Department of General Surgery, Nanjing Drum Tower Hospital, The Affiliated Hospital of Nanjing University Medical School, Nanjing, Jiangsu 210008, China; Tel: +86 256818 2099; Fax: +86 2568182097 or +86 1585050 2391; Email: guan-wx@163.com; Min Feng, MD, PhD, Department of General Surgery, Nanjing Drum Tower Hospital, The Affiliated Hospital of Nanjing University Medical School, Nanjing, Jiangsu 210008, China; Tel: +86 256818 2099; Fax: +86 256818 2097; Email: fengmindoctor@163.com
}

(MMPs), play an important role in the pathological and physiological processes of cancer development [6]. MMPs and their tissue inhibitors (TIMPs) were found to be associated with gastric cancer development. For example, MMP2 is implicated in the effects of $\mathrm{p} 21$-activated protein kinase 1 on the invasion of gastric cancer cells [7], while isocitrate dehydrogenase 2 inhibited gastric cancer cell invasion by decreasing the level of MMP7 [8]. Clinical studies showed that MMP9/13 play critical roles in the incidence and prognosis of gastric cancer $[9,10]$. In addition, MMP1/3/10/12/14 and TIMP1/2 were all reported to be associated with the development of gastric cancer [11-17].

Proliferation is another important biological feature associated with the prognosis of cancer. Alcohol was found to promote colorectal cancer proliferation by leading to hypermethylation of ADHFE1[18]. However, the role of alcohol in gastric cancer proliferation remains unclear [19]. Our previous studies indicated that alcohol consumption might result in endoplasmic reticulum stress (ER-stress) [20]. The ER is the site of protein folding, modification, assembly and intracellular calcium storage. An ER-stressrelated inflammatory response can occur via nuclear factorkappa B (NF- $\mathrm{B})$ and C-jun amino terminal kinase (JNK)/ transcription factor activator protein 1 (AP-1) [21,22]. Chronic inflammation is associated with the occurrence, proliferation and metastasis of tumors, suggesting that alcohol-induced ER stress may lead to inflammation and the subsequent proliferation and metastasis of cancer cells [23].

In this study, we investigated the effects of alcohol on the invasion and proliferation of gastric cancer. 


\section{Materials and Methods Cell culture}

Human gastric cancer cell lines (AGS and HGC-27) and human gastric mucosal endothelial cells (GES-1) were purchased from the Shanghai Institute of Cell Biology (Chinese Academy of Sciences, Shanghai, China). Cells were cultured in Dulbecco's modified Eagle's medium (DMEM) supplemented with 10\% FBS, $100 \mathrm{U} / \mathrm{ml}$ penicillin and $100 \mu \mathrm{g} / \mathrm{ml}$ streptomycin (all from Gibco, Gaithersburg, $\mathrm{MD}, \mathrm{USA}$ ) at $37^{\circ} \mathrm{C}$ in a humidified atmosphere with $5 \%$ $\mathrm{CO}$ 2. Cell lines were cultured with ethanol at $0 \mathrm{mmol} / \mathrm{L}, 35$ $\mathrm{mmol} / \mathrm{L}$, or $85 \mathrm{mmol} / \mathrm{L}$ in serum-containing culture medium for $24 h$.

\section{Invasion and cell migration assay}

The effects of alcohol on the invasion of cancer cells was determined using a modified Boyden chamber invasion assay [24]. Cells were cultured to $80 \%$ confluency and serum-starved overnight. The cells were then trypsinized and a viable cell count was obtained. Homogeneous single cell suspensions $\left(1 \times 10^{5}\right.$ cells/well $)$ were added to the upper chambers in serum-free medium containing alcohol (0/35/85 mmol/L) and/or GM6001 (GM, $0.2 \mathrm{nmol} / \mathrm{L}$, MCE, Monmouth Junction, NJ, USA). An equal volume of the same medium containing $10 \%$ FBS was placed in the lower chamber (the well beneath the filter) to act as a chemoattractant. The assay plates were incubated at $37^{\circ} \mathrm{C}$ for $24 \mathrm{~h}$. Following incubation, the filters were fixed with $3 \%$ glutaraldehyde in phosphate-buffered saline and stained with crystal violet for cell quantification.

\section{Quantitative real-time reverse transcription-polymerase chain reaction (qRT-PCR)}

Total RNA was isolated from each cell line with the RNApure tissue kit (Thermo Fisher Scientific, Inc. Waltham, MA, USA) following the manufacturer's instructions. The expression levels of mRNA for MMP2/7/9/13 and GAPDH were determined by qRTPCRwith an ABI Prism 7900HT (Applied Biosystems). Values were calculated as n-fold differences in gene expression relative to GAPDH. The following sets of primers were used in the PCR amplification: GAPDH (forward, 5' GAA GGT GAA GGT CGG AGT 3'and reverse, 5' GAA GAT GGT GAT GGG ATT TC 3'); MMP2 (forward, 5' GAG AAC CAA AGT CTG AAG AG 3'and reverse, 5' GGA GTG AGA ATG CTG ATT AG 3'); MMP7 (forward, 5'GAGTGAGCTACAGTGGGAACA 3' and reverse, 5' CTATGACGCGGGAGTTTAACAT 3'), MMP9 (forward, 5' GGG AGA CGC CCA TTT CG 3' and reverse, 5' CGC GCC ATC TGC GTT T 3'), MMP13 (forward, 5' CAG ACT TCA CGA TGG CAT TGC 3' and reverse, 5' AGG AAA AGC ATG AGC CAG CA 3’ ).

\section{Western blot analysis}

Whole cell lysates were prepared as described previously [20]. Total protein concentrations were determined using a bicinchoninic acid protein assay kit (KeyGen, Jiangsu, China). Equal amounts $(30 \mu \mathrm{g})$ of protein extracted from the cultured cells were run on $10 \%$ SDS-PAGE, followed by electro-transfer to polyvinylidene fluoride membranes (Millipore, Bedford, MA, USA). The membranes were blocked with 5\% skim milk powder for $2 \mathrm{~h}$. Then, the membranes were incubated overnight at $4^{\circ} \mathrm{C}$ with the primary antibodies, anti-GAPDH, antiCyclin D, anti-PCNA, anti-MMP2, anti-MMP7, antiMMP9 and anti-MMP13 (all from Abcam, USA), then with horseradish peroxidase-conjugated secondary antibody goat anti-rabbit IgG (ZSGB-Bio, Beijing, China) for $2 \mathrm{~h}$ at room temperature. The membranes were visualized by chemiluminescence (Millipore) and exposed with an automatic exposure machine (Bio-Rad, USA) in a dark room, and then Quantity One software was used for grayscale analysis.

\section{Animal model}

Male BALB/C nude mice (18-20g) were obtained from SLAC Laboratory Animal Co., Ltd. (Shanghai, China). All animals were treated according to the guidelines for the use of experimental animals and were approved by the Institutional Animal Care and Research Advisory Committee at Nanjing University (Nanjing, China). Briefly, HGC-27 cells $\left(5 \times 10^{5}\right.$ cells/mouse $)$ were subcutaneously transplanted into the axillary area of nude mice. The mice were housed in a SPF barrier environment after the completion of feeding. Tumor nodules $(0.3 \times 0.3 \mathrm{~cm})$ were observable in all of the mice with naked eye after a week. At that time, the mice started to receive water containing ethanol $(85 \mathrm{mmol} / \mathrm{L})$ by oral gavage, which continued for three weeks. Thereafter, the tumor-bearing mice were sacrificed, tumor tissues were isolated, and the weights and volumes of the tumors were determined. The tumor volume was measured by a Vernier caliper and calculated as [length $\left.\times(\text { width })^{2}\right] / 2$.

\section{Statistical analysis}

Data are presented as the means $\pm \mathrm{SD}$. For the statistical analysis of differences between two groups, Student's t-test for independent means was used. A value of $P<0.05$ was considered to be statistically significant, and a $P$-value $<$ 0.01 was considered to be very significant. The statistical analysis was performed using the SPSS 19.0 software.

\section{Results}

The baseline expression of MMPs was elevated in gastric cancer cells

We examined the MMP2/7/9/13mRNA and protein expression levels in two cancer lines (AGS and HGC27) and non-malignant gastric mucosal cells (used as a control; GES-1). It was found that MMP2 was more highly expressed in HGC-27 cells, while the MMP7/9/13 protein 
levels showed no significant differences compared with the GES-1cells (Figure 1A and B). In AGS cells, the protein level of MMP7, but not MMP2/9/13, was significantly elevated compared with the GES-1cells (Figure 1A and B). The transcription levels of MMPs were almost consistent with protein levels, but the MMP2 mRNA level was significantly increased in HGC-27 cells, but not AGS cells (Figure 1C).

\section{Alcohol treatment promoted MMPs expression in gastric cancer cells}

To determine the effects of alcohol on MMPs expression in gastric cancer cells, we treated the AGS and HGC27 cells with different concentrations of alcohol and then determined the MMP2/7/9/13 protein and mRNA levels. We found that the protein levels of MMP2/7 were increased in AGS cells after alcohol treatment (35 mM and $85 \mathrm{mM}$ ), while the protein levels of MMP9/13 were significantly increased only when exposed to the higher concentration of alcohol (85 mM). In HGC-27 cells, the MMP7/9/13 protein levels were increased after exposure to both concentrations of alcohol. The MMP2 protein level was increased only after treatment with the higher concentration of alcohol (Figure 2A and B).With regard to the transcriptional levels, the MMP2/7/9 expression levels were increased in AGC cells after exposure to both concentrations of alcohol, while the MMP13 mRNA level was increased only after treatment with the higher concentration of alcohol. The mRNA levels of MMP2/7/9/13 in the HGC-27 cells were increased by both concentrations of alcohol (Figure 2C).

\section{Alcohol treatment promoted the invasion of gastric cancer cells via MMPs}

MMPs play a critical role in cancer cell invasion $[7,8]$. To evaluate the effects of alcohol on gastric cancer cell invasion, we selected HGC-27 cells for an invasion experiment given their stronger response to alcohol stimulation noted above. We found that alcohol treatment promoted gastric cancer cell invasion in a concentrationdependent manner (Figure 3). To clarify the role of MMPs in the effects of alcohol on gastric cancer cell invasion, GM6001 was used to neutralize MMPs. GM6001 is a broad-spectrum MMPs inhibitor that inhibits the activity of MMPs-1/2/3/7/8/9/12/14/26 [25]. We found that GM6001 suppressed the invasion of cancer cells and prevented the stimulatory effects of alcohol (Figure 3).

\section{Ethanol exposure stimulated the proliferation of gastric cancer cells}

Proliferation is an important biological feature associated with cancer development [26]. We found that the protein levels of Cyclin D and PCNA, two markers of cell proliferation $[11,12]$, were increased in HGC-27 cells after alcohol treatment (35 $\mathrm{mM}$ and $85 \mathrm{mM}$ ), while in the AGS
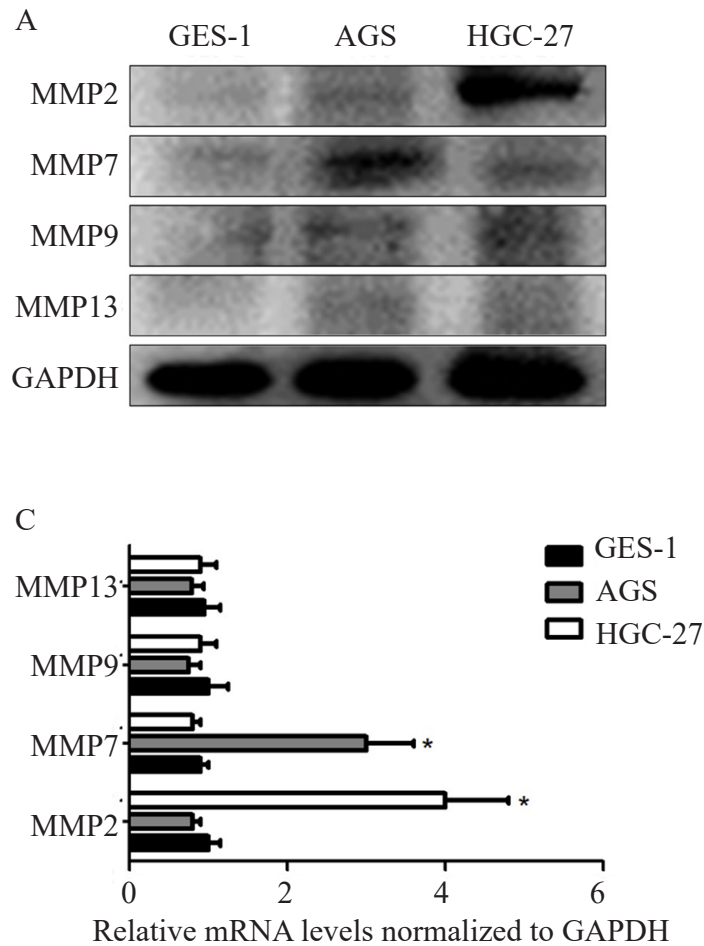

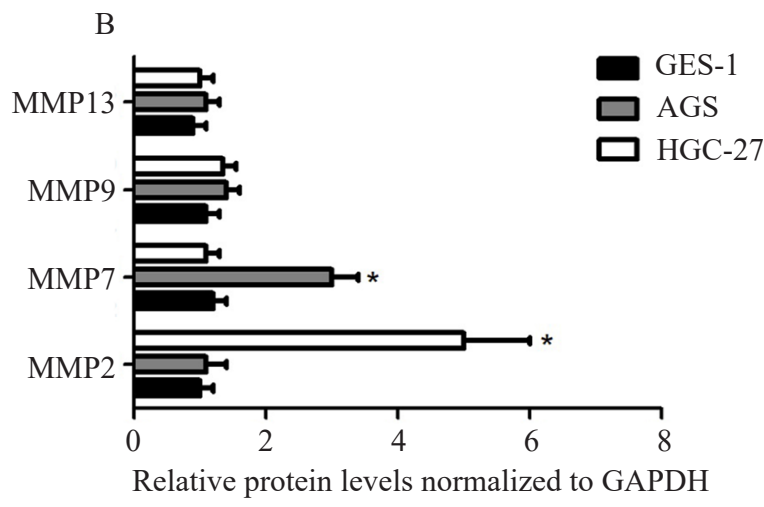

Figure 1 The expression of MMPs is elevated in gastric cancer cells. (A and B) The protein expression levels of MMP2, MMP7, MMP9 and MMP13 in GES-1, AGS and HGC-27 cells were determined by Western blot. (C) The mRNA levels of MMP2, MMP7, MMP9 and MMP13 in GES-1, AGS and HGC-27 cells were determined by qRT-PCR. The results were normalized to GAPDH. Data were expressed as the means \pm SD.n $=5, * P<0.05$. 
cell line, alcohol stimulation only resulted in a significant increase in the Cyclin D protein level (Figure 2D).

\section{Alcohol consumption promoted the growth of heterotopic gastric cancer tumors in mice}

To further understand the effects of alcohol on gastric cancer proliferation, we carried out an in vivo study. In the in vivo study, HGC-27 cells were used to establish an animal heterotopic tumor transplantation model as described in the Materials and Methods section. Alcohol treatment (via oral gavage to simulate normal consumption) promoted gastric cancer tumor growth, with increased tumor volumes and weights compared to the controls administered water (Figure 4).

\section{Discussion}

Alcohol use or abuse is a leading risk factor for various diseases. A recent study indicated that the global adult alcohol consumption per-capita increased from $5.9 \mathrm{~L}$ in 1990 to $6.5 \mathrm{~L}$ in 2017 , and would increase to $7.6 \mathrm{~L}$ by 2030 [27]. Epidemiological studies have found that alcohol was associated with the occurrence and prognosis of gastric
A

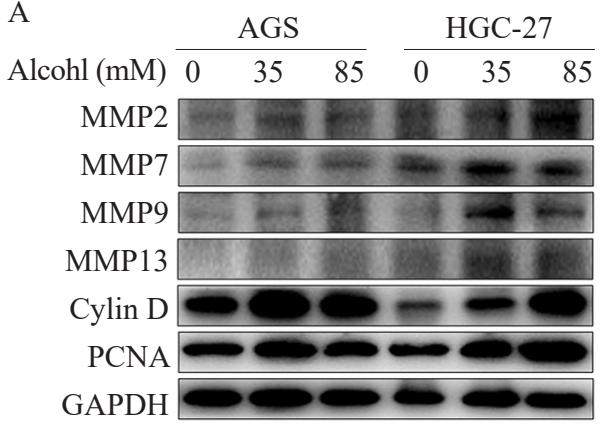

C

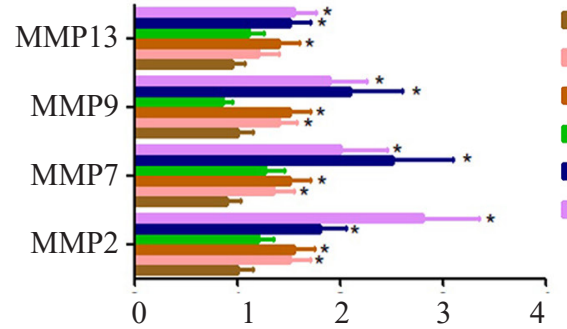

Relative Protein Levels Normalized to GAPDH
$\mathrm{B}$

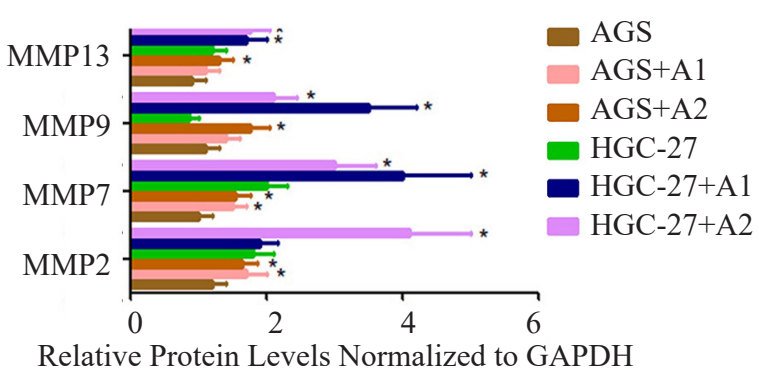

$\mathrm{D}$

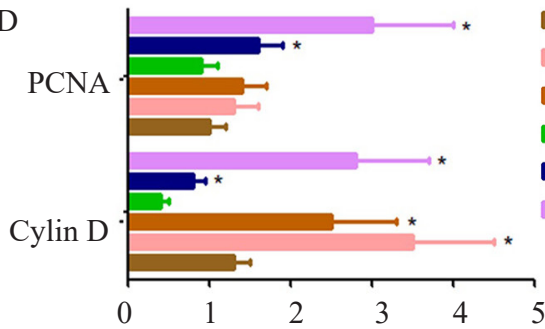

AGS

AGS+A1

$\mathrm{AGS}+\mathrm{A} 2$

HGC-27

- $\mathrm{HGC}-27+\mathrm{A} 1$

HGC-27+A2

Relative Protein Levels Normalized to GAPDH

Figure 2 Alcohol promotes MMPs, Cyclin D and PCNA expression. (A and B) AGS and HGC-27 cells were treated with different concentrations of alcohol, then the protein expression levels of MMP2, MMP7, MMP9 and MMP13 were determined by Western blot. (C) The mRNA levels of MMP2, MMP7, MMP9 and MMP13 were determined by qRT-PCR. The results were normalized to GAPDH. Data were expressed as the means \pm SD. $\mathrm{n}=5$, * $P<0.05$. A1, alcohol treatment $(35 \mathrm{mM})$; A2, alcohol treatment $(85 \mathrm{mM})$.
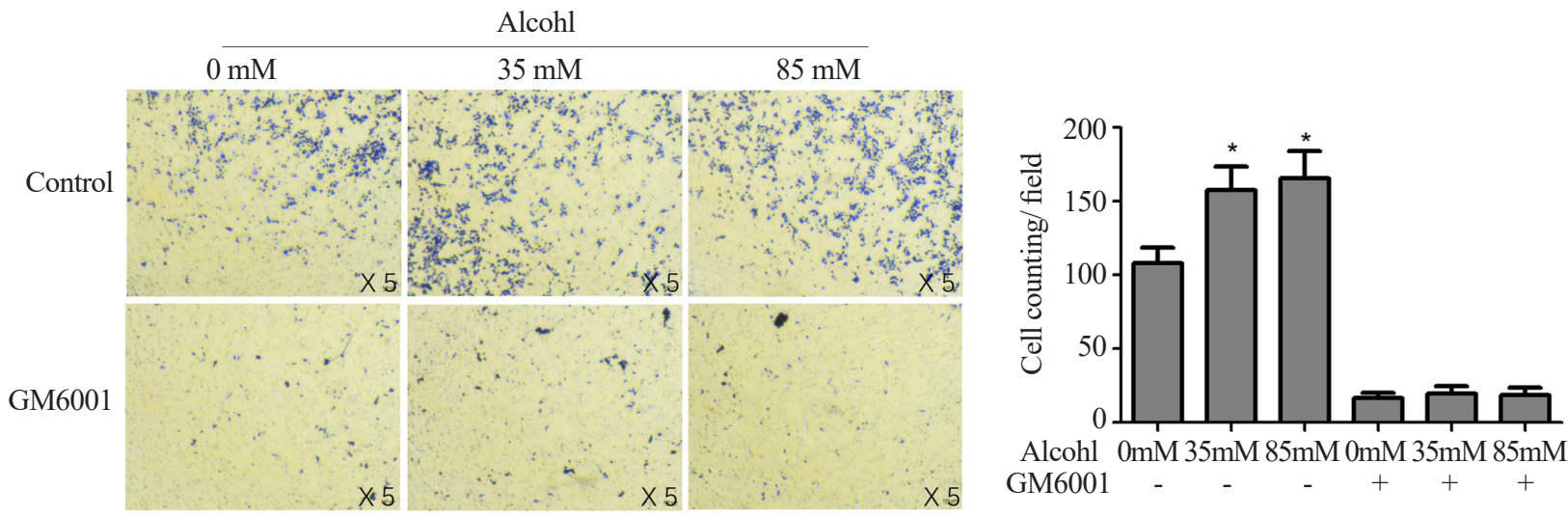

Figure 3 Alcohol promotes the invasion of gastric cancer cells. HGC-27 cells were plated into an invasion assay system as described in the Materials and Methods. After a $24 \mathrm{~h}$ incubation, the filters were stained with crystal violet for quantification of the cells. Data were expressed as the means \pm SD. $\mathrm{n}=3, * P<0.05$. 

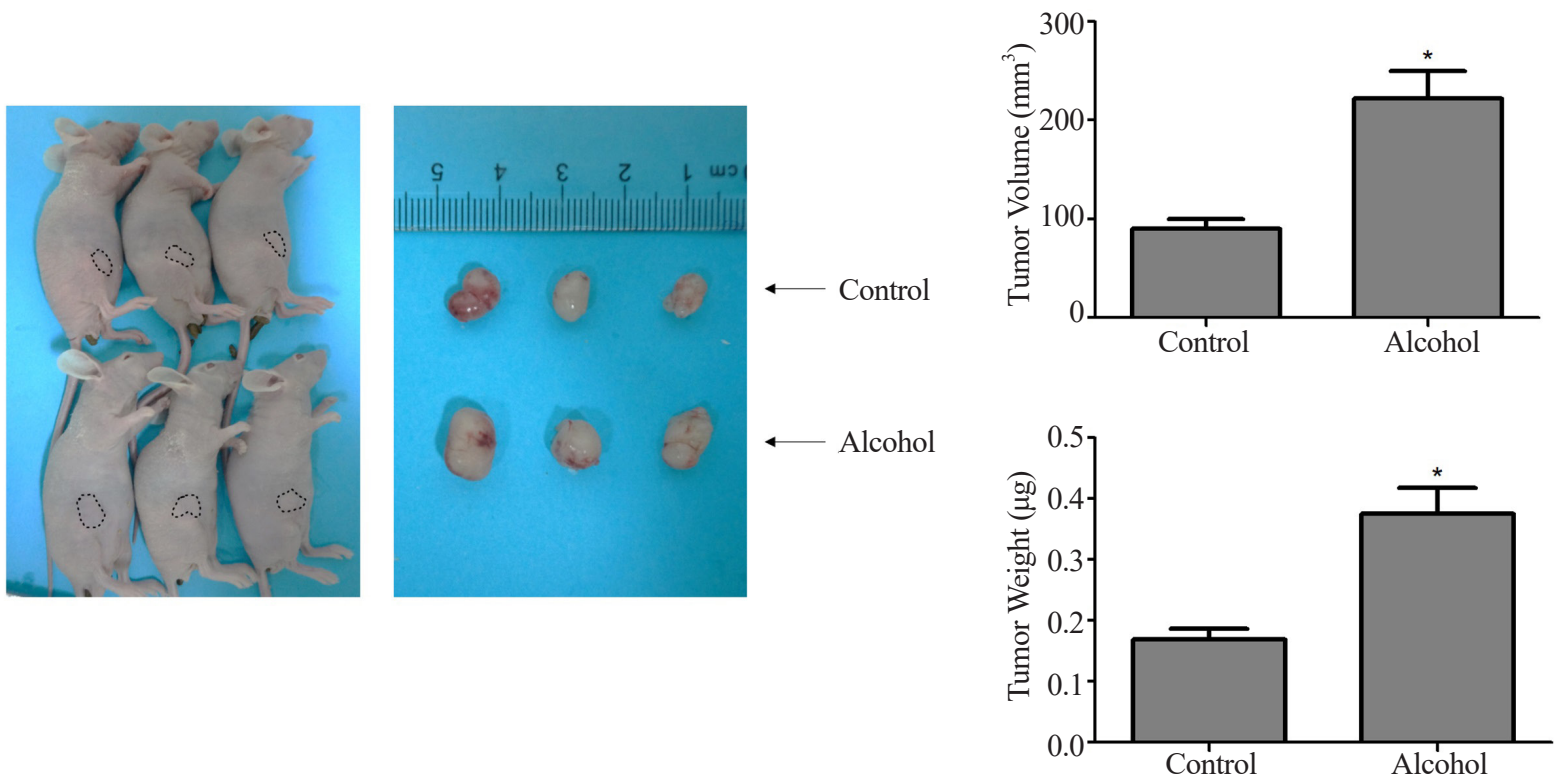

Figure 4 Alcohol consumption promotes gastric cancer tumor growth. HGC-27 cells were used to establish a heterotopic transplantation animal tumor model. The animals were sacrificed after 3 weeks of alcohol treatment $(85 \mathrm{mM})$. The volume and weight of tumors were measured and compared with controls. Data were expressed as the means $\pm \mathrm{SD}$. $\mathrm{n}=3, * P<0.05$.

cancer $[3,4]$, while the underlying mechanism by which alcohol promotes gastric cancer development remains unclear. In this study, we investigated the role of alcohol in the invasion and proliferation of gastric cancer.

MMPs degrade the proteins in the ECM and increase tumor invasion and metastasis. MMP2/7 were reported to play a critical role in gastric cancer invasion [7,8], while MMP9/13 were found to be related to the prognosis of gastric cancer $[9,10]$. Our previous study demonstrated that MMP9/13 were associated with colon cancer metastasis [28].In this study, we found that the baseline MMP2 expression was increased in HGC-27 cells and the MMP7 level was increased in AGS cells, although MMP9/13 did not show any significant elevation in either of the cancer cell lines compared with GES-1 cells.

Alcohol consumption was reported to increase the occurrence of gastric cancer and lead to a worse prognosis in clinical studies [3]. In this study, we found that alcohol treatment promoted MMP2/7/9/13 expression in both AGS and HGC-27 cells. However, the degree of MMPs elevation was different in the two cell lines. The response of HGC-27 cells to alcohol stimulation in MMPs expression was stronger, so we selected these cells for the invasion experiment. We found that alcohol promoted the invasion of gastric cancer cells in a concentration-dependent manner.

In this study, we used GM6001 to confirm the role of MMPs in gastric cancer cell invasion. It was found that GM6001 significantly suppressed HGC-27 invasion and decreased the effects of alcohol. These data indicate that alcohol exposure promotes gastric cancer invasion by increasing MMPs expression.
Proliferation is associated with tumor development and prognosis [26]. In this study, we found that the Cyclin D level was increased in both HGC-27 and AGS cells after alcohol treatment. The PCNA level was also increased, but only in HGC-27 cells. We used the HGC-27 cell line to establish a heterotopic transplantation animal tumor model. It was found that alcohol consumption promoted gastric cancer tumor growth, with increased tumor volume and weight.

\section{Conclusion}

In conclusion, this study demonstrated that alcohol promotes gastric cancer invasion and proliferation. The elevation of MMPs after alcohol treatment contributed to the invasion of the cancer cells. Although the underlying mechanism(s) by which alcohol consumption promotes gastric cancer proliferation remain unclear, this study provides information on how ethanol may lead to gastric cancer development and progression, and provides a stepping stone for further studies.

\section{Conflict of Interests}

The authors of this manuscript declare no conflicts of interest.

\section{Funding}

This work was supported by the National Nature Science Foundation of China (81670561), the Nature Science Foundation of Jiangsu Province (QNRC2016022), the Fundamental Research Funds for the Central Universities (021414380445), and the Nature Science Foundation of Nanjing (ZKX14022). 


\section{References}

1. Bray F, Ferlay J, Soerjomataram I, Siegel RL, Torre LA, Jemal A. Global cancer statistics 2018: GLOBOCAN estimates of incidence and mortality worldwide for 36 cancers in 185 countries. CA Cancer J Clin 2018;68(6):394-424.

2. Zheng R, Sun K, Zhang S, Zeng H, Zou X, Chen R, Gu X, Wei W, He J. Report of cancer epidemiology in China, 2015. Zhonghua Zhong Liu Za Zhi 2019;41(1):19-28.

3. Minami Y, Kanemura S, Oikawa T, Suzuki S, Hasegawa Y, Miura K, Nishino Y, Kakugawa Y, Fujiya T. Associations of cigarette smoking and alcohol drinking with stomach cancer survival: a prospective patient cohort study in Japan. Int J Cancer 2018;143(5):1072-85.

4. Kim SA, Choi BY, Song KS, Park CH, Eun CS, Han DS, Kim YS, Kim HJ. Prediagnostic smoking and alcohol drinking and gastric cancer survival: a Korean prospective cohort study. Korean J Gastroenterol 2019;73(3):141-51

5. Eble JA, Niland $\mathrm{S}$. The extracellular matrix in tumor progression and metastasis. Clin Exp Metastasis 2019;36(3):171-98.

6. Han YP. Matrix metalloproteinases, the pros and cons, in liver fibrosis. J Gastroenterol Hepatol 2006;21 Suppl 3: S88-91.

7. Li LH, Wu GY, Lu YZ, Chen XH, Liu BY, Zheng MH, Cai JC. p21-activated protein kinase 1 induces the invasion of gastric cancer cells through c-Jun NH2-terminal kinase-mediated activation of matrix metalloproteinase-2. Oncol Rep 2017;38(1):193-200.

8. Wu D. Isocitrate dehydrogenase 2 inhibits gastric cancer cell invasion via matrix metalloproteinase 7. Tumour Biol 2016;37(4): 5225-30.

9. Okada R, Naito M, Hattori Y, Seiki T, Wakai K, Nanri H, Watanabe M, Suzuki S, Kairupan TS, Takashima N, Mikami H, Ohnaka K, Watanabe Y, Katsuura-Kamano S, Kubo M, Hamajima N, Tanaka H. Matrix metalloproteinase 9 gene polymorphisms are associated with a multiple family history of gastric cancer. Gastric Cancer 2017;20(2):246-53.

10. Sheibani S, Mahmoudian RA, Abbaszadegan MR, Chamani J, Memar B, Gholamin M. Expression analysis of matrix metalloproteinase-13 in human gastric cancer in the presence of Helicobacter Pylori infection. Cancer Biomark 2017;18(4):349-56.

11. Peng Q, Xu Y. Association between promoter polymorphisms of matrix metalloproteinase-1 and risk of gastric cancer. Onco Targets Ther 2015;8:2519-26.

12. Cao J, Li Z, Yang L, Liu C, Luan X. Association between tissue inhibitor of metalloproteinase- 3 gene methylation and gastric cancer risk: a meta-analysis. Genet Test Mol Biomarkers 2016;20(8):427-31.

13. Jiang H, Zhou Y, Liao Q, Ouyang H. Helicobacter pylori infection promotes the invasion and metastasis of gastric cancer through increasing the expression of matrix metalloproteinase-1 and matrix metalloproteinase-10. Exp Ther Med 2014;8(3):769-74.

14. Zheng J, Chu D, Wang D, Zhu Y, Zhang X, Ji G, Zhao H, Wu G, Du J, Zhao Q. Matrix metalloproteinase-12 is associated with overall survival in Chinese patients with gastric cancer. J Surg Oncol 2013;107(7):746-51.
15. Naseh G, Mohammadifard M. Upregulation of cyclin-dependent kinase 7 and matrix metalloproteinase-14 expression contribute to metastatic properties of gastric cancer. IUBMB Life 2016;68(10):799-805.

16. Grunnet M, Mau-Sorensen M, Brunner N. Tissue inhibitor of metalloproteinase 1 (TIMP-1) as a biomarker in gastric cancer: a review. Scand J Gastroenterol 2013;48(8):899-905.

17. Lukaszewicz-Zajac M, Mroczko B, Guzinska-Ustymowicz K, Pryczynicz A, Gryko M, Kemona A, Kedra B, Szmitkowski M. Matrix metalloproteinase 2 (MMP-2) and their tissue inhibitor 2 (TIMP-2) in gastric cancer patients. Adv Med Sci 2013;58(2):235-43.

18. Moon JW, Lee SK, Lee YW, Lee JO, Kim N, Lee HJ, Seo JS, Kim J, Kim HS, Park SH. Alcohol induces cell proliferation via hypermethylation of ADHFE1 in colorectal cancer cells. BMC Cancer 2014;14:377.

19. Xu M, Floyd HS, Greth SM, Chang WC, Lohman K, Stoyanova R, Kucera GL, Kute TE, Willingham MC, Miller MS. Perillyl alcohol-mediated inhibition of lung cancer cell line proliferation: potential mechanisms for its chemotherapeutic effects. Toxicol Appl Pharmacol 2004;195(2):232-46.

20. Kao E, Shinohara M, Feng M, Lau MY, Ji C. Human immunodeficiency virus protease inhibitors modulate $\mathrm{Ca} 2+$ homeostasis and potentiate alcoholic stress and injury in mice and primary mouse and human hepatocytes. Hepatology 2012;56(2):594-604.

21. Zhou R, Yazdi AS, Menu P, Tschopp J. A role for mitochondria in NLRP3 inflammasome activation. Nature 2011;469(7329):221-5.

22. Garg AD, Kaczmarek A, Krysko O, Vandenabeele P, Krysko DV, Agostinis P. ER stress-induced inflammation: Does it aid or impede disease progression? Trends Mol Med 2012;18(10):589-98.

23. Wessler S, Krisch LM, Elmer DP, Aberger F. From inflammation to gastric cancer - the importance of Hedgehog/GLI signaling in Helicobacter pylori-induced chronic inflammatory and neoplastic diseases. Cell Commun Signal 2017;15(1):15.

24. Zheng L, Li D, Xiang X, Tong L, Qi M, Pu J, Huang K, Tong Q. Meth$\mathrm{yl}$ jasmonate abolishes the migration, invasion and angiogenesis of gastric cancer cells through down-regulation of matrix metalloproteinase 14. BMC Cancer 2013;13:74.

25. Bohm G, Groll J, Heffels KH, Heussen N, Ink P, Alizai HP, Neumann UP, Schnabel R, Mirastschijski U. Influence of MMP inhibitor GM6001 loading of fibre coated polypropylene meshes on wound healing: Implications for hernia repair. J Biomater Appl 2018(10);32:134359.

26. Li D, Hu C, Li H. Survivin as a novel target protein for reducing the proliferation of cancer cells. Biomed Rep 2018;8(5):399-406

27. Manthey J, Shield KD, Rylett M, Hasan OSM, Probst C, Rehm J. Global alcohol exposure between 1990 and 2017 and forecasts until 2030: a modelling study. Lancet 2019; 393(10190):2493-502.

28. Wang Q, Feng M, Yu T, Liu X, Zhang P. Intratumoral regulatory T cells are associated with suppression of colorectal carcinoma metastasis after resection through overcoming IL-17 producing T cells. Cell Immunol 2014;287(2):100-5. 